Running head: $\quad$ PCS in populations with and without mTBI

Post-Concussion Syndrome: Prevalence after mild traumatic brain injury in comparison with a sample without head injury

Philip J. A. Dean, Darragh O’Neill and Annette Sterr Department of Psychology, University Of Surrey, Guildford, UK

Correspondence to: Philip Dean, Department Of Psychology, University Of Surrey, Guildford, Surrey, UK, GU2 7XH

Fax: +44 (0)1483 682914; Phone: +44 (0)1483 682877; e-mail: p.dean@ @urrey.ac.uk

Keywords: Mild Traumatic Brain Injury, Persistent Post-Concussion Syndrome, Rivermead Post-Concussion Symptoms Questionnaire, Base Rates. 


\begin{abstract}
Primary Objective: To compare the prevalence of persistent post-concussion syndrome (PCS;

$>1$ year post-injury) in participants with mild traumatic brain injury (mTBI) and those without head injury.
\end{abstract}

Research Design: Cross-sectional sample of 119 participants with mTBI and 246 without previous head injury.

Methods: Online questionnaires collected data about post-concussion symptoms, cognitive failures, anxiety, depression, sleep behaviour and post-traumatic stress disorder. Variability within the sample was addressed by splitting by PCS diagnosis to create four groups: mTBI+PCS, mTBI-PCS, Control+PCS and Control-PCS. PCS was diagnosed using ICD-10 criteria in all groups, with controls not requiring previous head injury.

Main Outcomes and Results: PCS was present to a similar extent in participants with no head injury $(34 \%)$ compared to those with mTBI $(31 \%)$. Only report of headaches, which could be caused by expectation bias, distinguished between mTBI+PCS and Control+PCS groups. In addition, significantly higher cognitive problems were observed in participants with mTBI compared with the control group

Conclusions: Persistent PCS, as currently defined, is not specific to mTBI. These data suggest that somatic and cognitive symptoms are most likely to be able to distinguish PCS after mTBI from that present in the general population. Further research is necessary into these factors in order to create more specific PCS diagnostic criteria. 


\section{Post-Concussion Syndrome: Prevalence after mild traumatic brain injury in comparison with a sample without head injury \\ Introduction}

The majority (70-90\%) of all-severity traumatic brain injury (TBI) can be classified as mild (mTBI; [1]), and its overall prevalence within a hospital setting is estimated at 0.1 to $0.3 \%$ [2]. However, only a proportion of individuals with mTBI are admitted to hospital when visiting the emergency department (around 10-25\%) [3, 4]. Furthermore, emergency department data is likely to underestimate the incidence of mTBI, as it does not capture those individuals who do not seek or receive medical attention [5]. A range of somatic, affective and cognitive symptoms are often induced after an mTBI, such as headaches, feelings of depression and poor memory [6]. These symptoms are collectively known as PostConcussion Syndrome (PCS; [7]) or Post-Concussional Disorder (PCD; [8]). Symptoms usually occur days after the initial injury and resolve within around 3 months [9-16].

Nevertheless, persistent PCS symptoms (> 3 months after mTBI, [17]) have also been observed, with some participants in earlier research continuing to report symptoms a year or more after injury [18-22]. The onset of these symptoms has been shown to vary post-injury, with increased or novel reporting of symptoms seen at follow-up compared to initial (acute) testing $[18,23]$. Approximately $5 \%$ of those reporting to hospital with an mTBI subsequently experience persistent PCS [24]. As the majority of those with mTBI are not admitted to hospital $[3,4]$, it is likely that this figure underestimates the true incidence of persistent PCS. Therefore, it is clear that persistent PCS remains a significant public health problem.

The main diagnostic criteria for PCS, the International Classification of Diseases (ICD-10; [7]) and the Diagnostic and Statistical Manual of Mental Disorders (DSM-IV; [8]), have differing viewpoints on how to determine the longer lasting problems associated with the injury. Symptoms in three or more categories are required to diagnose PCS by both criteria; 
ICD-10 lists 16 symptoms in 6 categories, whereas DSM-IV lists 13 in 8 categories. The symptoms lists are similar, but the way they are divided into categories differs substantially. For example, in the ICD-10 criteria, headache, dizziness and fatigue are in the same category, so can only be counted once for diagnostic purposes. DSM-IV criteria list these three symptoms in different categories. Therefore, if you have only these three symptoms, PCS diagnosis is possible with DSM-IV, but not ICD-10 criteria. One further difference is that DSM-IV criteria require objective neuropsychological tests demonstrating cognitive difficulty, whereas ICD-10 stipulates only subjective evidence. Some studies have investigated how these two criteria differ [25-28], with significant differences seen in the diagnosis of PCS within the same dataset. A comparison of ICD-10 and DSM-IV criteria along with individual symptoms concluded that the differences between these criteria were due to the use of objective cognitive testing, minimum symptom duration and clinical significance in the DSM-IV [25]. Diagnosis on the basis of symptoms only leads to similar incidence of PCS for both criteria. However, neither criteria set is seen as preferable, with each having its own problems [28].

Part of the difficulty in diagnosing PCS, and the inherent ambiguity of the current diagnostic criteria, is the non-specificity of the symptoms. Depression [29-31], chronic pain [32], post-traumatic stress (PTSD; [33]), stress [34], anxiety [35], fatigue [36], involvement in litigation [37, 38], gender [39], and a number of other factors [13, 21, 40-46], have been shown to influence the levels of reported PCS symptoms, even without the presence of mTBI $[29,32,37]$. Indeed, it has been suggested that pre-existing psychological factors [47] as well as the subjective nature of the necessarily self-reported data $[46,48]$ can have an influence on PCS symptom levels. Furthermore, postconcussion-like symptoms have been reported in the general population [49-53] at levels that would result in PCS diagnosis in a population with head injury. 
It is these ambiguities and complications in PCS diagnosis that this current study aims to explore, particularly the incidence of postconcussion-like symptoms in the general population. A comprehensive investigation into postconcussion-like symptoms and related variables (depression, anxiety, PTSD, cognitive failure, daytime sleepiness, litigation, chronic pain and gender) was carried out in healthy control participants and this was compared to persistent PCS (>1 year post-injury [17]) levels in a group with mTBI.

A major limitation in PCS research is that variable diagnosis and inconsistent control measures have made synthesis of research studies difficult [54]. A review of the persistent PCS literature [17] indicated that idiographic differences between symptomatic participants tend to be hidden under total group effects, especially when limited to a particular chronicity. Individual variations in damage sustained and recovery progression mean that sampling participants within a specific chronicity (e.g. 3 months) could result in a fairly heterogeneous group and mask any injury-related changes. Therefore, our study sample ranges from those who had an mTBI 1 year previously to those whose injury occurred many years ago.

In addition, splitting study groups by PCS diagnosis, including the control (non-mTBI) group [53, 55], to look at co-variables and neuropsychological tests has been suggested as a useful future direction [17]. A few studies have adopted this method [15, 21, 28, 40, 42]. Out of these, only a couple of studies $[21,40]$ examined persistent PCS symptoms at least a year post injury, and they neglected to split either participants with mTBI [40] or those in the control group [21] by PCS diagnosis and postconcussion-like symptom report. While nonhead injured participants cannot by definition have PCS, assessment of the symptoms comprising this syndrome in these participants is valid and potentially informative. The current study aims to examine the specificity of this symptom combination to individuals with mTBI. Consequently, the extent to which participants with and without mTBI exceeded the criteria threshold for PCS diagnosis is assessed in the current research. Control 
participants were categorised as having PCS, for analysis purposes only, when they met all the ICD-10 criteria except the caveat of previous head injury. This grouping provides a means of determining the clinical meaningfulness and specificity of PCS diagnosis, as well as directly comparing the occurrence of its constituent symptoms in individuals with and without mTBI.

Recruitment was deliberately focused on members of the public in order to eliminate the referral bias that can occur in hospital based studies [3, 4], particularly in studies that recruit patients referred for ongoing clinical symptoms. The majority of individuals who experience an mTBI are not admitted to hospital, and prevalence of mTBI in the general population is greater than that seen in emergency departments or hospitals. This suggests that a large number of those who sustain an mTBI are unreported in the traditional literature $[5,56]$, although they may subsequently experience persistent, worsening or even emerging PCS symptoms. For this reason, we used an online survey to collect the data, ensuring that a more representative sampling of PCS in both populations with mTBI and those without head injury was achieved. As inclusion in the survey does not require the participant to have had an mTBI or experienced symptoms, or to think they might have, it enables a more complete sampling of the full spectrum of mTBI, broadening the focus beyond the more obvious hospital-treated cases. A comparison of ICD-10 and modified DSM-IV [57, 58] PCS diagnosis criteria was conducted in order to clarify diagnostic variability and the generalisability of research findings.

\section{Method}

\section{Recruitment}

In order to obtain a representative, cross-sectional, sample of participants with mild traumatic brain injury (mTBI), including those who have not reported to hospital, recruitment 
was achieved via advertisements placed in public places around Guildford. These advertisements gave the web address of an online survey. To encourage participation, completed surveys were entered into a prize draw with three possible cash prizes. In addition to demographic information, respondents were asked whether they had ever experienced an injury to the head. If participants responded positively, then a series of further questions were asked in order to determine whether the injury met the diagnosis criteria for mTBI. Participants were also screened for previous major head injury; 12 participants (mTBI+PCS: 8; mTBI-PCS: 4) were excluded on the basis of this criterion. 350 members of the public, chiefly comprising university staff and students, were included in the subsequent analysis. The study protocol was given a favourable opinion by the University Ethics Committee.

\section{Diagnosis}

mTBI was determined using ICD-10 criteria [59]. In order to meet these criteria, participants had to report one or more of the following: dizziness or confusion; loss of consciousness (LOC) for 30 minutes or less; post-traumatic amnesia (PTA) for less than 24 hours. A description of the injury, the date of injury, and a report of any previous minor head injury were also taken to aid diagnosis.

PCS diagnosis employed ICD-10 criteria [7]. The number and severity of symptoms were collected using the Rivermead Post-Concussion symptoms Questionnaire (RPQ; [60-62]), as in previous studies $[11,14,21,39,40,53,63]$. PCS was diagnosed if participants reported that three (or more) of the symptom categories listed in the ICD-10 criteria were more of a problem after the head injury.

For participants in the control (no history of head injury) group, a modified Rivermead Post-concussion symptoms Questionnaire for Controls (RPQ-C; [21]) was used to assess symptoms. In the usual RPQ, the question "Compared with before the accident, do you now (i.e. over the last 24 hours) suffer from:" is used. The RPQ-C changed this to "Compared 
with your peers, do you now (i.e. over the last 24 hours) suffer from:". Diagnosis was achieved in the same way for participants in the control group as those in the mTBI group, with the exception that for controls there was no "history of head trauma". This allows direct comparison of symptom levels, and diagnostic specificity, in the two populations.

\section{Study Sample}

Out of the 350 respondents, 106 were deemed to have had an mTBI, and 244 were found to have no mTBI history. These two groups were divided further on the basis of PCS diagnosis into: participants with mTBI and PCS (mTBI+PCS; n=33), mTBI and no PCS (mTBI-PCS; n=73), PCS but no mTBI (Control+PCS, $n=83$ ) and no mTBI or PCS (Control-PCS; $n=161$ ). More detailed demographics for each of these are shown in Table 1.

\section{Questionnaires}

In addition to the RPQ, questionnaires were included in the survey on everyday cognitive failures (Cognitive Failures Questionnaire, CFQ; [64]), post-traumatic stress disorder (PTSD; Impact of Event Scale-Revised: IES-R; [65]); daytime sleepiness (Epworth Sleepiness Scale: ESS; [66, 67]), anxiety and depression (Hospital Anxiety and Depression Scale: HADS; [68]). In addition, demographic information (Table 1) was collected on age, gender, medication, other neurological conditions, dyslexia, chronic pain, and involvement in litigation.

\section{$R P Q-C$}

The modified form of the RPQ for controls, the RPQ-C [21], changes the initial question from a within-person comparison ("Compared with before the accident, do you now (i.e. over the last 24 hours) suffer from:") to a between-person comparison ("Compared with your peers, do you now (i.e. over the last 24 hours) suffer from:"). To ensure that this difference does not impact on the diagnostic equivalence across the RPQ and RPQ-C, we conducted a repeat assessment, one year after initial RPQ-C assessment. A subsample of the control group 
was retested using either the RPQ-C or a newly-developed within-person comparison version of the RPQ-C (RPQ-C-W). The RPQ-C-W employed the following question format: "Compared with one year ago, do you now (i.e. over the last 24 hours) suffer from:". 48 participants in the control group completed the repeat assessment: 26 participants responded to the RPQ-C-W, and 22 responded to the initial RPQ-C.

\section{Other PCS Diagnostic Criteria}

As with previous studies [25-28], we tested the effect of different diagnostic criteria on our dataset. As our data was collected using an online survey, it was not possible to obtain neuropsychological test data. However, previous studies have used modified DSM-IV PCS diagnostic criteria which are employed in the absence of neuropsychological test data [57, 58]. In order to make the modified DSM-IV criteria (mDSM-IV) more analogous to full DSM-IV criteria (but in the absence of neuropsychological test data), we also used novel diagnostic criteria where participants must meet mDSM-IV criteria and also report at least one cognitive problem (poor memory, poor concentration, taking longer to think). This was called the mDSM-IV(COG). A comparison of the effect of ICD-10, mDSM-IV and mDSMIV(COG) criteria on PCS diagnosis was performed.

\section{Statistical Analyses}

Demographics presented as percentages in Table 1 were analysed for sample differences using chi-square $\left(\chi^{2}\right)$ tests, while standardised residuals $(z)$ were calculated as a post-hoc method of identifying more precisely the source of any significant differences. Independent $t$ -

tests were performed on age, IES-R and time since injury. $\chi^{2}$ tests were also conducted to compare the relative numbers of people with PCS within the mTBI and Control groups. A series of two-way between-subjects analyses of variance (ANOVA) tests were carried out to examine differences within and interaction between the independent variables of mTBI (2 levels: presence/absence of mTBI) and PCS (2 levels: presence/absence of PCS) on the four 
psychometric measures employed in this study: RPQ, CFQ, HADS and ESS. A further series of two-way between-subjects ANOVA tests were conducted on the 16 items of the RPQ with the same independent variables. Where significant interactions between the mTBI and PCS factors were established, independent $t$-tests were carried out as a simple main effects analysis and suitable correction for the familywise error rate was applied. The advantage of this analysis approach over the more commonly used table of symptom intensities is that it enables any statistical interaction at the test item level between PCS diagnosis and mTBI to be quantitatively assessed.

Cochran's $Q$ tests were performed to look for differences in PCS diagnosis (ICD-10, mDSM-IV and mDSM-IV(COG)) for mTBI and control groups separately. This was followed by post-hoc McNemar $\chi^{2}$ tests with correction for the familywise error rate, to examine diagnostic equivalence across the RPQ-C and RPQ-C-W in the repeat testing of controls.

\section{Results}

\section{Demographics}

Significant differences (Table 1) were found for age $(F(3,346)=5.49, p=0.001)$, gender $\left(\chi^{2}(3, N=350)=8.23 ; p=0.041\right)$, medication $\left(\chi^{2}(3, N=350)=22.34 ; p<0.001\right)$ and dyslexia $\left(\chi^{2}(3, N=350)=9.96 ; p=0.019\right)$. Post-hoc tests revealed that the participants in the mTBI groups were significantly older than participants in the Control+PCS (mTBI+PCS: mean difference $=4.8$ years, $p=0.035$; mTBI-PCS: mean difference $=4.0$ years, $p=0.02$ ) and Control-PCS groups (mTBI+PCS: mean difference $=4.3$ years, $p=0.046$; $\mathrm{mTBI}-\mathrm{PCS}$ : mean difference $=3.5$ years, $p=0.02)$. There were more male participants $(z=2.0, p=$ $0.046)$ and participants with dyslexia $(z=2.0, p=0.046)$ in the mTBI-PCS group compared to all other groups. More participants in the mTBI+PCS group took some kind of medication $(z=3.8 ; p<0.001)$ compared to all other groups. In addition, post-traumatic stress disorder 
(PTSD, as measured by IES-R sum score) was present to a greater degree in the mTBI+PCS group compared to the mTBI-PCS group $(t(40)=3.68, p=0.001)$.

---Insert Table 1 about here---

Although there was no significant difference in education level between groups, $73 \%$ of the study sample was educated to a degree level or above, which is a much higher proportion than the general population (20\%) [69].

\section{PCS specificity to $\mathrm{mTBI}$}

The proportion of individuals experiencing PCS (Figure 1) was not statistically different $\left(\chi^{2}\right.$ $(1, N=350)=0.28 ; p=0.60)$ between mTBI $(33 / 106$ or $31 \%)$ and control participants $(83 / 244 ; 34 \%)$.

---Insert Figure 1 about here---

\section{PCS co-variables}

The ANOVAs revealed participants with mTBI had significantly higher scores on the CFQ $(F(1,346)=11.00, p=0.001)$ compared to controls (Figure 2$)$. There was also a trend for higher scores on the RPQ $(F(1,346)=3.10, p=0.079)$. No significant differences were observed on the ESS or HADS. However, participants with PCS had higher scores on all four measures $(\operatorname{RPQ}(F(1,346)=288.00, p<0.001)$; CFQ $(F(1,346)=40.97, p<0.001)$; HADS-Anxiety $(F(1,346)=48.42, p<0.001) ; \operatorname{HADS}-$ Depression $(F(1,346)=53.71, p<$ $0.001)$; ESS $(F(1,346)=5.80, p=0.017))$ when compared to those without PCS. No significant interaction was seen between mTBI and PCS for any of the scales.

---Insert Figure 2 about here---

\section{RPQ Item Analysis}


The most apparent aspect of the profile plot of mean item scores (Figure 3) is the similarity between the mTBI+PCS and Control+PCS and between the mTBI-PCS and Control-PCS groups across each of the symptoms assessed by the measure.

\section{---Insert Figure 3 about here---}

A series of ANOVA analyses were conducted to examine group differences for each item. Unsurprisingly, participants with PCS scored significantly higher $(p<0.001)$ on each item, regardless of whether they have experienced an mTBI. Nonetheless, differences were observed between participants with and without mTBI.

Those with mTBI had significantly higher scores for headaches $(F(1,346)=21.71, p<$ $0.001)$, dizziness $(F(1,346)=14.37, p<0.001)$, nausea $(F(1,349)=3.92 ; p=0.048)$, taking longer to think $(F(1,346)=4.52 ; p=0.034)$, light sensitivity $(F(1,346)=7.46, p=$ $0.007)$ and double vision $(F(1,346)=6.21, p=0.013)$. A significant interaction between PCS and mTBI was observed for headaches $(F(1,346)=5.72, p=0.017)$. Following up this interaction, it was found that the mTBI+PCS group scored higher on the headaches item $(t(114)=3.90, p<0.001)$ than the Control+PCS group. A comparison of the mTBI-PCS and Control-PCS groups also demonstrated that they differed significantly on the headaches item $(t(232)=2.11, p=0.036)$.

\section{Diagnostic Reliability and Validity}

There was no significant difference $\left(\operatorname{McNemar} \chi^{2}(1, N=26)=1.29 ; p=0.453\right)$ in the proportion of participants in the control group defined as having PCS after completing the RPQ-C-W compared to when the same participants carried out the initial RPQ-C one year previously. Participants who completed the RPQ-C for a second time showed identical 
proportions of PCS compared to their initial RPQ-C response one year previously (McNemar $\left.\chi^{2}(1, N=22)=0 ; p=1.0\right) .{ }^{1}$

Upon following ICD-10 criteria, 31\% $(n=33)$ of the mTBI group were diagnosed with PCS, and 34\% ( $n=83)$ of the control group were diagnosed with PCS. mDSM-IV criteria diagnosed $34 \%(n=36)$ of the mTBI group and 33\% $(n=80)$ of the control group as having PCS. Finally, mDSM-IV (COG) criteria diagnosed PCS in $26 \%(n=28)$ of the mTBI group, and $25 \%(n=61)$ of the control group.

\section{---Insert Figure 4 about here---}

The proportions of participants (Figure 4) diagnosed as having PCS were statistically different between the three diagnoses for both participants with mTBI (Cochran's $Q=7.00 ; p$ $=0.030$ ) and those with no prior head injury (Cochran's $Q=21.90 ; p<0.001$ ). Post-hoc tests, corrected for multiple comparisons, revealed that the mDSM-IV (COG) criteria diagnosed proportionally fewer participants in the Control+PCS group than both ICD-10 $\left(\operatorname{McNemar} \chi^{2}(1)=20.17 ; p<0.001\right)$ and mDSM-IV $($ McNemar $=19.0 ; p<0.001)$ criteria. For participants with mTBI, it was again the mDSM-IV (COG) that accounted for the difference, diagnosing fewer cases of PCS than the mDSM-IV criteria only $(\mathrm{McNemar}=8.0$; $p=0.008)$.

\section{Discussion}

\section{Principal findings}

This study confirmed earlier reports of high levels of persistent PCS in participants with mTBI [19-22] and a notable incidence rate of postconcussion-like symptoms in a non-clinical

\footnotetext{
1 Equivalence of the RPQ and RPQ-C was further examined through a multidimensional scaling analysis of the two measures and no notable difference in latent structure was observed. Additional details on this latter analysis are available from the first author.
} 
population [49-53]. Significantly, it also established that postconcussion-like symptoms are present to such a degree that diagnosis of PCS is similar in a self-selected sample of persons with and without mTBI (Figure 1). Combined with the detailed analysis of the data discussed below, this suggests that PCS (as currently defined) is associated with, but not specific to, mTBI $[23,53,70]$. This is important, as the symptoms used to define PCS must be specific enough to distinguish from populations without head injury (i.e. the Control+PCS population) in order to be useful as a diagnostic tool.

\section{Co-Variables}

Analysis of co-variables such as depression, anxiety, daytime sleepiness and cognitive failures (Figure 2) revealed that scores on such constructs increase significantly with PCS diagnosis, a finding that is consistent with previous results [21, 29, 34, 42]. However, there were significant differences between participants with brain injury, regardless of PCS diagnosis, and healthy controls. Participants with mTBI had significantly more cognitive failures than those with no history of head injury. In addition, there was a trend for greater post-concussion symptom report in participants with mTBI $(p=0.079)$. This suggests that while the overall diagnosis of PCS did not differ, there are some symptoms which are able to distinguish between the groups. If the current PCS diagnosis criteria are not specific enough to mTBI, it may be worth adjusting the criteria to give more weight to the more discriminatory symptoms. Indeed, previous research has suggested that the symptoms constituting PCS (as recorded by the RPQ) may show greater reliability and validity id split into Research has already been conducted into the test-retest reliability Comparison between the groups with mTBI revealed that those with PCS were also more likely to have PTSD than those without PCS, as reported by others $[33,71]$. This will be discussed in further detail in the RPQ Analysis and Implications sections. 
Of the other co-variables represented in Table 1, participants with mTBI were older than control participants. The difference in age between the youngest and oldest group was only 4.8 years, and is consequently unlikely to have contributed notably to any group differences. Participants in the mTBI-PCS group were more likely to have dyslexia (8\%), and had proportionally more males than all other groups. Female gender is associated with PCS symptoms [39], and the data presented here confirm that, for mTBI participants, those with PCS are more likely to be female. Participants with mTBI and PCS were more likely to be on medication (52\%) than all other participant groups (Control+PCS: 23\%; mTBI-PCS: 19\%, Control-PCS: 15\%). The difference between the two participant groups with PCS is particularly interesting, as it implies that symptoms after head injury require increased medication and not simply high report of related symptoms. Higher medication use in the mTBI+PCS group was predominantly through prescription drugs for depression, anxiety, pain, and migraines. This seems to be at odds with the data showing no difference in depression or anxiety between those with PCS and those without, suggesting that subjective reporting of these symptoms does not effectively identify their severity. A possible explanation is that the hypochondriacal concern mentioned in ICD-10 criteria for PCS [7] makes participants with mTBI more likely to seek out medication since they have an event to attribute their symptoms to. Use of migraine medication is consistent with the finding that headaches are an important point of discrimination. However, it remains unknown whether these medications were used to treat symptoms prior to injury and not those caused by the mTBI.

\section{RPQ Analysis}

Analysis of the mean score profile plot (Figure 3) revealed that participants with mTBI had greater average scores for the majority of the somatic (headaches, dizziness, nausea, light 
sensitivity, and double vision) and one of the cognitive symptoms (taking longer to think). However, interaction between mTBI and PCS was only seen for "headaches". Further analysis of the four subgroups revealed that the mTBI+PCS group reported more headaches on average than the Control+PCS group. The same was true for the mTBI-PCS group compared to the Control-PCS group. Therefore, although the other somatic and cognitive symptoms distinguished between participants with and without head injury as a whole, only report of headaches discriminates between mTBI participants with PCS and control participants with PCS. This was also demonstrated in an earlier study [71], where the sample data was adjusted to account for PTSD and depression. Before adjustment, a number of PCS symptoms differentiated between participants with and without brain injury. After adjustment, the groups only differed in their report of headaches, which has been demonstrated to be highly prevalent after brain injury [72]. It is necessary to distinguish between participants with and without head injury in order to diagnose PCS as something separate from symptoms present in the general population. Although symptoms of headache are not specific to head injury, the presence of headaches is the symptom most directly related to head injury, and therefore most expected to occur post-injury. Perhaps its prevalence here is merely due to an expectation bias [73, 74].

The other symptoms that classify more generally between participants with and without head injury require further research to increase their diagnostic usefulness. In particular, cognitive differences seem promising as they are seen both in the RPQ item analysis and in the CFQ sum score differences. Combining symptom report of headaches with criteria based on the other symptoms that more generally classify mTBI may create a more precise definition and diagnosis of PCS.

Comparison of RPQ item report to previous studies is difficult, as few have looked at persistent PCS. One study [14] compared item report from 3 months and 12 months post 
injury. It reported similar proportions of PCS diagnosis in populations with mTBI as seen in this study, and a strikingly similar profile of RPQ item report from 3 months post injury. However, it contained no control data or data from participants with mTBI more than one year post injury, and so is only a partial replication of the findings.

\section{Diagnostic Reliability}

In order to verify the levels of PCS observed within this healthy non-head-injured population, we undertook some additional analyses. The wording of the RPQ-C was tested to make sure that the postconcussion-like symptom levels reported were not the result of the wording of the questionnaire. Changing the wording from a between-person comparison ("Compared to your peers") to a within-person comparison ("Compared to one year ago") did not change the number of control subjects meeting PCS using ICD-10 diagnostic criteria. In addition, the symptoms of participants in the control group who re-took the RPQ-C did not differ from their initial report. Both re-tests were performed a year after the participants initially filled out the RPQ-C. Therefore, the levels of PCS observed in control groups are reliable and stable over time.

Previous research has found that ICD-10 criteria diagnose greater numbers of participants with PCS than DSM-IV criteria in the same mTBI dataset [25-28]. Consequently, it is possible that the elevated levels of PCS determined for the control population in this dataset were due to ICD-10 diagnostic criteria. As such, we set out to determine whether diagnostic criteria had an effect on our control populations, as well as those with mTBI. Due to the nature of survey methodology, it was not possible to carry out objective cognitive testing, and therefore a modified set of DSM-IV (mDSM-IV) criteria $[57,58]$ for use with controls as well as individuals with mTBI was employed. In addition, an mDSM-IV criterion in which at least one subjective cognitive complaint must be reported (mDSM-IV (COG)) was also 
introduced. This allows a diagnosis closer to the original DSM-IV, as it accounts for cognitive problems as much as is possible in a survey setting. The mDSM-IV (COG) diagnosed fewer participants with PCS in participants without head injury compared to both the other scales, and fewer compared to the mDSM-IV in participants with mTBI. This suggests that the cognitive testing element of the typical DSM-IV accounts for the lower levels of PCS diagnosed [25-28]. Using ICD-10 criteria did increase the prevalence of PCS in participants without head injury compared to when using mDSM-IV (COG) criteria. The use of objective cognitive testing, as per usual DSM-IV criteria may further aid differentiation between control and MTBI populations. However, even with mDSM-IV (COG) criteria there was still a significant proportion $(25 \%)$ of participants without head injury exhibiting symptoms equivalent to PCS. A further caveat is that cognitive symptoms such as memory and concentration problems have been shown to vary after injury, with novel and increased reporting of these symptoms observed with repeated testing [18, 23]. Factors unrelated to brain injury may be contributing to this variability in cognitive symptoms, especially so many years post-injury.

\section{Implications}

The data presented in this study have implications for both clinical and research practice, as they demonstrate that the intensity and pattern of symptoms and co-factors of PCS are very similar between participants with and without mTBI. It raises the issue of diagnosis in a clinical setting, and whether the current definition of PCS is valid in classifying persistent PCS. Distinguishing symptoms caused by the injury from baseline symptoms is difficult. If the levels of PCS are the same after mTBI as in a normal population, it could be that we are just measuring naturally-occurring pre-existing symptoms that have subsequently been attributed to the head injury. Indeed, some previous papers have suggested that there is an 
attribution bias, in the sense that participants expect to have particular symptoms [73, 74] or overestimate their level of functioning before the injury [75]. Another possibility is that the similarity in the PCS levels, and symptom profile, between participants with and without mTBI is due to the gradual attenuation of reported symptoms to a degree where it no longer differs greatly from the non-head injured population. Participants sustaining an mTBI a year or more previously could then attribute their residual symptoms to the injury, where in fact the symptoms they experience also appear in the healthy population. Most research on this topic has been done on the acute phase after mTBI where the majority of individuals become symptom free around 3 months after injury [9, 11]. Long term outcome in PCS several years after mTBI has not been looked at in the same detail $[20,21,40]$, and it would be interesting to see if others find the same result.

PCS symptom report was associated with greater levels of PTSD, depression, anxiety and cognitive failures in participants with mTBI and PCS. Previous studies that have adjusted for co-variables have observed very few PCS symptom report differences between groups with and without head injury [71]. Therefore, it could be that PCS symptom report is purely related to these other psychological factors, and not to the initial mTBI.

Researchers must also consider whether grouping participants in a control group together when analysing data can produce different results. Certainly, if you group either participants with mTBI or those with no head injury together without thinking of PCS diagnosis, the within-group variance may be larger than the between-group variance, meaning that you wash out potential differences between the groups [17].

\section{Limitations and future research}

An important issue raised by these data, which was not possible to determine with the current study design, is how the symptoms reported now relate to those before, or 
immediately after the injury. This sort of analysis is difficult to attain, but essential to answering whether the similarity in symptoms reported years after injury to the occurrence of these symptoms in the general population is due to the non-specificity of the criteria for PCS or an attenuation of initially more intense problems. There are however, some sport-related research paradigms that are beginning to help distinguish these factors by conducting prospective studies with baseline symptoms and cognitive performance before injury [76-78]. Participants in the study had higher than average levels of education, and may therefore not be entirely representative of the general population. Attempts were made to recruit more extensively from the local area, however it must be acknowledged that pattern of PCS symptom report may vary in populations with differing educational level. Indeed, some studies suggest that lower educational level may lead to greater persistent PCS report [79], which may mean that this current study underestimates the real incidence of PCS. Another limitation of the current study was the small number of participants that completed the alternative RPQ-C, potentially limiting the statistical power of that particular analysis.

The design of this study, which relied on subjective self-report data rather than objective measures, also precluded a thorough comparison of the differing diagnostic PCS criteria as full DSM-IV criteria could not be met. However, mDSM-IV (COG) criteria diagnosed fewer control participants with PCS, and there were higher cognitive failures and more cognitive RPQ items reported by those with mTBI than those without. This suggests that cognitive deficit could be an important distinguishing factor for PCS between those with concussion and healthy non brain injured populations $[42,80]$. Subjective criteria may not be specific enough to diagnose PCS after mTBI. Objective criteria, such as cognitive testing, may be more exacting in their diagnosis. It is not possible to explicitly test this hypothesis with the current survey methodology, although it does offer present potential for future research. Cognitive complaints are observed frequently after mTBI [22]. Differences in cognitive 
performance assessed through objective measures have also been reported [81-85], but are difficult to replicate, especially a year or more after the injury [21]. One reason for this could be the variability in report of cognitive symptom after injury [18, 23]. Alternatively, it could be due to the grouping together of those with and without PCS in both participants with mTBI, and those with no previous head injury. As previously stated [17], and confirmed in the current study, grouping data together in this way can create heterogeneous groups and hence is likely to attenuate potential differences. Objective cognitive testing of the four groups presented here could potentially clarify whether there are cognitive deficits associated with mTBI and persistent PCS.

\section{Conclusions}

In summary, postconcussion-like symptoms are seen in healthy participants to such a degree that diagnosis of PCS is at similar levels to those who have had an mTBI. Although there is evidence of some differences between these two groups, the majority of co-factors are strikingly similar. This could be due to PCS, as currently defined, not being specific enough to mTBI. Alternatively, it could suggest that the symptoms experienced by participants with mTBI a long time after injury have returned to normal levels. There are implications for clinical diagnosis, as well as research design, where a case for screening participants in the control group for PCS could be argued. Future studies will explore objective cognitive testing as a distinguishing factor between participants with mTBI and those in the control group, using the four groups presented here. We hope to find that subdividing the populations in this way leads to less ambiguous conclusions. 


\section{Acknowledgements}

We would like to thank Dr. Ellen Seiss, Dr. Katherine Herron and Dr. Adam McNamara for their help and advice during the collection of this data.

\section{Declaration of Interest}

This study was supported by a Wingate Foundation Scholarship, UK. 


\section{References}

1. Cassidy JD, Carroll LJ, Peloso PM, Borg J, von Holst H, Holm L, Kraus J, Coronado VG. Incidence, risk factors and prevention of mild traumatic brain injury: results of the WHO Collaborating Centre Task Force on Mild Traumatic Brain Injury. J Rehabil Med 2004(43):28-60.

2. Carroll LJ, Cassidy JD, Holm L, Kraus J, Coronado VG. Methodological issues and research recommendations for mild traumatic brain injury: the WHO Collaborating Centre Task Force on Mild Traumatic Brain Injury. J Rehabil Med 2004(43):113-25.

3. Sosin DM, Sniezek JE, Thurman DJ. Incidence of mild and moderate brain injury in the United States, 1991. Brain Inj 1996;10(1):47-54.

4. Bazarian JJ, McClung J, Shah MN, Cheng YT, Flesher W, Kraus J. Mild traumatic brain injury in the United States, 1998--2000. Brain Inj 2005;19(2):85-91.

5. National Center for Injury Prevention and Control. Report to Congress on Mild Traumatic Brain Injury in the United States: Steps to Prevent a Serious Public Health Problem. . Atlanta, GA: Centers for Disease Control and Prevention; 2003.

6. Ryan LM, Warden DL. Post concussion syndrome. Int Rev Psychiatry 2003;15(4):310-6.

7. World Health Organisation. International Statistical Classification of Diseases and Related Health Problems. Geneva, Switzerland: World Health Organisation; 1992.

8. American Psychological Association. Diagnosis and Statistical Manual of Mental Disorders. Washington, DC: American Psychiatric Association; 1994.

9. Ingebrigtsen T, Waterloo K, Marup-Jensen S, Attner E, Romner B. Quantification of post-concussion symptoms 3 months after minor head injury in 100 consecutive patients. J Neurol 1998;245(9):609-12. 
10. Korinthenberg R, Schreck J, Weser J, Lehmkuhl G. Post-traumatic syndrome after minor head injury cannot be predicted by neurological investigations. Brain Dev 2004;26(2):113-7.

11. Lannsjo M, af Geijerstam JL, Johansson U, Bring J, Borg J. Prevalence and structure of symptoms at 3 months after mild traumatic brain injury in a national cohort. Brain Inj 2009;23(3):213-9.

12. Lundin A, de Boussard C, Edman G, Borg J. Symptoms and disability until 3 months after mild TBI. Brain Inj 2006;20(8):799-806.

13. Ponsford J, Willmott C, Rothwell A, Cameron P, Kelly AM, Nelms R, Curran C, Ng K. Factors influencing outcome following mild traumatic brain injury in adults. J Int Neuropsychol Soc 2000;6(5):568-79.

14. Sigurdardottir S, Andelic N, Roe C, Jerstad T, Schanke AK. Post-concussion symptoms after traumatic brain injury at 3 and 12 months post-injury: a prospective study. Brain Inj 2009;23(6):489-97.

15. Sroufe NS, Fuller, D.S., West, B.T., Singal, B.M., Warchausky, S.A. and Maio, R.F. A Prospective, Longitudinal Study Of Post-Concussive Symptoms and Neurocognitive Function anfter Mild Traumatic Brain Injury in Children 10-17 Years Of Age. Pediatrics 2008.

16. Yang CC, Hua MS, Tu YK, Huang SJ. Early clinical characteristics of patients with persistent post-concussion symptoms: a prospective study. Brain Inj 2009;23(4):299306.

17. Bigler ED. Neuropsychology and clinical neuroscience of persistent post-concussive syndrome. J Int Neuropsychol Soc 2008;14(1):1-22.

18. Dikmen S, Machamer J, Fann JR, Temkin NR. Rates of symptom reporting following traumatic brain injury. J Int Neuropsychol Soc 2010;16(3):401-11. 
19. Hessen E, Anderson V, Nestvold K. MMPI-2 profiles 23 years after paediatric mild traumatic brain injury. Brain Inj 2008;22(1):39-50.

20. Killam C, Cautin RL, Santucci AC. Assessing the enduring residual neuropsychological effects of head trauma in college athletes who participate in contact sports. Arch Clin Neuropsychol 2005;20(5):599-611.

21. Sterr A, Herron K, Hayward C, Montaldi D. Are mild head injuries as mild as we think? Neurobehavioral concomitants of chronic post-concussion syndrome. BMC Neurol 2006;6(1):7.

22. Stulemeijer M, Vos PE, Bleijenberg G, van der Werf SP. Cognitive complaints after mild traumatic brain injury: things are not always what they seem. J Psychosom Res 2007;63(6):637-45.

23. Meares S, Shores EA, Taylor AJ, Batchelor J, Bryant RA, Baguley IJ, Chapman J, Gurka J, Marosszeky JE. The prospective course of postconcussion syndrome: the role of mild traumatic brain injury. Neuropsychology 2011;25(4):454-65.

24. Iverson GL. Outcome from mild traumatic brain injury. Curr Opin Psychiatry 2005;18(3):301-17.

25. Boake C, McCauley SR, Levin HS, Contant CF, Song JX, Brown SA, Goodman HS, Brundage SI, Diaz-Marchan PJ, Merritt SG. Limited agreement between criteriabased diagnoses of postconcussional syndrome. J Neuropsychiatry Clin Neurosci 2004;16(4):493-9.

26. Boake C, McCauley SR, Levin HS, Pedroza C, Contant CF, Song JX, Brown SA, Goodman H, Brundage SI, Diaz-Marchan PJ. Diagnostic criteria for postconcussional syndrome after mild to moderate traumatic brain injury. J Neuropsychiatry Clin Neurosci 2005;17(3):350-6. 
27. Kashluba S, Casey JE, Paniak C. Evaluating the utility of ICD-10 diagnostic criteria for postconcussion syndrome following mild traumatic brain injury. J Int Neuropsychol Soc 2006;12(1):111-8.

28. McCauley SR, Boake C, Pedroza C, Brown SA, Levin HS, Goodman HS, Merritt SG. Correlates of persistent postconcussional disorder: DSM-IV criteria versus ICD-10. J Clin Exp Neuropsychol 2008;30(3):360-79.

29. Iverson GL. Misdiagnosis of the persistent postconcussion syndrome in patients with depression. Arch Clin Neuropsychol 2006;21(4):303-10.

30. Suhr JA, Gunstad J. Postconcussive symptom report: the relative influence of head injury and depression. J Clin Exp Neuropsychol 2002;24(8):981-93.

31. Trahan DE, Ross CE, Trahan SL. Relationships among postconcussional-type symptoms, depression, and anxiety in neurologically normal young adults and victims of mild brain injury. Arch Clin Neuropsychol 2001;16(5):435-45.

32. Radanov BP, Dvorak J, Valach L. Cognitive deficits in patients after soft tissue injury of the cervical spine. Spine (Phila Pa 1976) 1992;17(2):127-31.

33. Bryant RA, Creamer M, O'Donnell M, Silove D, Clark CR, McFarlane AC. Posttraumatic amnesia and the nature of post-traumatic stress disorder after mild traumatic brain injury. J Int Neuropsychol Soc 2009;15(6):862-7.

34. Gouvier WD, Cubic B, Jones G, Brantley P, Cutlip Q. Postconcussion symptoms and daily stress in normal and head-injured college populations. Arch Clin Neuropsychol 1992;7(3):193-211.

35. Moore EL, Terryberry-Spohr L, Hope DA. Mild traumatic brain injury and anxiety sequelae: a review of the literature. Brain Inj 2006;20(2):117-32. 
36. Johansson B, Berglund P, Ronnback L. Mental fatigue and impaired information processing after mild and moderate traumatic brain injury. Brain Inj 2009;23(1314):1027-40.

37. Dunn JT, Lees-Haley PR, Brown RS, Williams CW, English LT. Neurotoxic complaint base rates of personal injury claimants: implications for neuropsychological assessment. J Clin Psychol 1995;51(4):577-84.

38. Lees-Haley PR, Fox DD, Courtney JC. A comparison of complaints by mild brain injury claimants and other claimants describing subjective experiences immediately following their injury. Arch Clin Neuropsychol 2001;16(7):689-95.

39. Preiss-Farzanegan SJ, Chapman B, Wong TM, Wu J, Bazarian JJ. The relationship between gender and postconcussion symptoms after sport-related mild traumatic brain injury. Pm R 2009;1(3):245-53.

40. Chan RC. How severe should symptoms be before someone is said to be suffering from post-concussion syndrome? An exploratory study with self-reported checklist using Rasch analysis. Brain Inj 2005;19(13):1117-24.

41. Kashluba S, Paniak C, Casey JE. Persistent symptoms associated with factors identified by the WHO Task Force on Mild Traumatic Brain Injury. Clin Neuropsychol 2008;22(2):195-208.

42. Landre N, Poppe CJ, Davis N, Schmaus B, Hobbs SE. Cognitive functioning and postconcussive symptoms in trauma patients with and without mild TBI. Arch Clin Neuropsychol 2006;21(4):255-73.

43. McCauley SR, Boake C, Levin HS, Contant CF, Song JX. Postconcussional disorder following mild to moderate traumatic brain injury: anxiety, depression, and social support as risk factors and comorbidities. J Clin Exp Neuropsychol 2001;23(6):792808. 
44. Meares S, Shores EA, Taylor AJ, Batchelor J, Bryant RA, Baguley IJ, Chapman J, Gurka J, Dawson K, Capon L and others. Mild traumatic brain injury does not predict acute postconcussion syndrome. J Neurol Neurosurg Psychiatry 2008;79(3):300-6.

45. Nolin P, Heroux L. Relations among sociodemographic, neurologic, clinical, and neuropsychologic variables, and vocational status following mild traumatic brain injury: a follow-up study. J Head Trauma Rehabil 2006;21(6):514-26.

46. Suhr JA, Gunstad J. "Diagnosis Threat": the effect of negative expectations on cognitive performance in head injury. J Clin Exp Neuropsychol 2002;24(4):448-57.

47. Greiffenstein FM, Baker JW. Comparison of premorbid and postinjury mmpi-2 profiles in late postconcussion claimants. Clin Neuropsychol 2001;15(2):162-70.

48. Nolin P, Villemure R, Heroux L. Determining long-term symptoms following mild traumatic brain injury: method of interview affects self-report. Brain Inj 2006;20(11):1147-54.

49. Chan RC. Base rate of post-concussion symptoms among normal people and its neuropsychological correlates. Clin Rehabil 2001;15(3):266-73.

50. Fear NT, Jones E, Groom M, Greenberg N, Hull L, Hodgetts TJ, Wessely S. Symptoms of post-concussional syndrome are non-specifically related to mild traumatic brain injury in UK Armed Forces personnel on return from deployment in Iraq: an analysis of self-reported data. Psychol Med 2009;39(8):1379-87.

51. Iverson GL, Lange RT. Examination of "postconcussion-like" symptoms in a healthy sample. Appl Neuropsychol 2003;10(3):137-44.

52. Sawchyn JM, Brulot MM, Strauss E. Note on the use of the Postconcussion Syndrome Checklist. Arch Clin Neuropsychol 2000;15(1):1-8.

53. Wang Y, Chan RC, Deng Y. Examination of postconcussion-like symptoms in healthy university students: relationships to subjective and objective 
neuropsychological function performance. Arch Clin Neuropsychol 2006;21(4):33947.

54. Pertab JL, James KM, Bigler ED. Limitations of mild traumatic brain injury metaanalyses. Brain Inj 2009;23(6):498-508.

55. Hanna-Pladdy B, Berry ZM, Bennett T, Phillips HL, Gouvier WD. Stress as a diagnostic challenge for postconcussive symptoms: sequelae of mild traumatic brain injury or physiological stress response. Clin Neuropsychol 2001;15(3):289-304.

56. Segalowitz SJ, Lawson S. Subtle symptoms associated with self-reported mild head injury. J Learn Disabil 1995;28(5):309-19.

57. Mittenberg W, Strauman S. Diagnosis of mild head injury and the postconcussion syndrome. J Head Trauma Rehabil 2000;15(2):783-91.

58. Vanderploeg RD, Curtiss G, Luis CA, Salazar AM. Long-term morbidities following self-reported mild traumatic brain injury. J Clin Exp Neuropsychol 2007;29(6):58598.

59. Holm L, Cassidy JD, Carroll LJ, Borg J. Summary of the WHO Collaborating Centre for Neurotrauma Task Force on Mild Traumatic Brain Injury. J Rehabil Med 2005;37(3):137-41.

60. Eyres S, Carey A, Gilworth G, Neumann V, Tennant A. Construct validity and reliability of the Rivermead Post-Concussion Symptoms Questionnaire. Clin Rehabil 2005;19(8):878-87.

61. King NS, Crawford S, Wenden FJ, Moss NE, Wade DT. The Rivermead Post Concussion Symptoms Questionnaire: a measure of symptoms commonly experienced after head injury and its reliability. J Neurol 1995;242(9):587-92.

62. Potter S, Leigh E, Wade D, Fleminger S. The Rivermead Post Concussion Symptoms Questionnaire: a confirmatory factor analysis. J Neurol 2006;253(12):1603-14. 
63. Chaput G, Giguere JF, Chauny JM, Denis R, Lavigne G. Relationship among subjective sleep complaints, headaches, and mood alterations following a mild traumatic brain injury. Sleep Med 2009;10(7):713-6.

64. Broadbent DE, Cooper PF, FitzGerald P, Parkes KR. The Cognitive Failures Questionnaire (CFQ) and its correlates. Br J Clin Psychol 1982;21:1-16.

65. Baumert J, Simon H, Gundel H, Schmitt C, Ladwig KH. The Impact of Event Scale-Revised: evaluation of the subscales and correlations to psychophysiological startle response patterns in survivors of a life-threatening cardiac event: an analysis of 129 patients with an implanted cardioverter defibrillator. J Affect Disord 2004;82(1):2941.

66. Johns MW. A new method for measuring daytime sleepiness: the Epworth sleepiness scale. Sleep 1991;14(6):540-5.

67. Miletin MS, Hanly PJ. Measurement properties of the Epworth sleepiness scale. Sleep Med 2003;4(3):195-9.

68. Bjelland I, Dahl AA, Haug TT, Neckelmann D. The validity of the Hospital Anxiety and Depression Scale. An updated literature review. J Psychosom Res 2002;52(2):6977.

69. Census 2001 Key Statistics - Rural and urban area classification 2004 KS13 Qualifications and students [Internet]. Office For National Satistics (UK). 2004 [cited 2011 Oct 12]. Available from http://www.ons.gov.uk/

70. Gunstad J, Suhr JA. Perception of illness: nonspecificity of postconcussion syndrome symptom expectation. J Int Neuropsychol Soc 2002;8(1):37-47.

71. Hoge CW, McGurk D, Thomas JL, Cox AL, Engel CC, Castro CA. Mild traumatic brain injury in U.S. Soldiers returning from Iraq. N Engl J Med 2008;358(5):453-63. 
72. Hoffman JM, Lucas S, Dikmen S, Braden CA, Brown AW, Brunner R, Diaz-Arrastia R, Walker WC, Watanabe TK, Bell KR. Natural history of headache after traumatic brain injury. J Neurotrauma 2011;28(9):1719-25.

73. Hahn RA. The nocebo phenomenon: concept, evidence, and implications for public health. Prev Med 1997;26(5 Pt 1):607-11.

74. Mittenberg W, DiGiulio DV, Perrin S, Bass AE. Symptoms following mild head injury: expectation as aetiology. J Neurol Neurosurg Psychiatry 1992;55(3):200-4.

75. Gunstad J, Suhr JA. "Expectation as etiology" versus "the good old days": postconcussion syndrome symptom reporting in athletes, headache sufferers, and depressed individuals. J Int Neuropsychol Soc 2001;7(3):323-33.

76. Iverson GL, Brooks BL, Collins MW, Lovell MR. Tracking neuropsychological recovery following concussion in sport. Brain Inj 2006;20(3):245-52.

77. Jantzen KJ, Anderson B, Steinberg FL, Kelso JA. A prospective functional MR imaging study of mild traumatic brain injury in college football players. AJNR Am J Neuroradiol 2004;25(5):738-45.

78. McCrea M, Guskiewicz K, Randolph C, Barr WB, Hammeke TA, Marshall SW, Kelly JP. Effects of a symptom-free waiting period on clinical outcome and risk of reinjury after sport-related concussion. Neurosurgery 2009;65(5):876-82; discussion 882-3.

79. Luis CA, Vanderploeg RD, Curtiss G. Predictors of postconcussion symptom complex in community dwelling male veterans. J Int Neuropsychol Soc 2003;9(7):1001-15.

80. Cicerone KD, Azulay J. Diagnostic utility of attention measures in postconcussion syndrome. Clin Neuropsychol 2002;16(3):280-9. 
81. Mathias JL, Beall JA, Bigler ED. Neuropsychological and information processing deficits following mild traumatic brain injury. J Int Neuropsychol Soc 2004;10(2):286-97.

82. McAllister TW, Sparling MB, Flashman LA, Guerin SJ, Mamourian AC, Saykin AJ. Differential working memory load effects after mild traumatic brain injury. Neuroimage 2001;14(5):1004-12.

83. O'Jile JR, Ryan LM, Betz B, Parks-Levy J, Hilsabeck RC, Rhudy JL, Gouvier WD. Information processing following mild head injury. Arch Clin Neuropsychol 2006;21(4):293-6.

84. Pare N, Rabin LA, Fogel J, Pepin M. Mild traumatic brain injury and its sequelae: characterisation of divided attention deficits. Neuropsychol Rehabil 2009;19(1):11037.

85. Pontifex MB, O'Connor PM, Broglio SP, Hillman CH. The association between mild traumatic brain injury history and cognitive control. Neuropsychologia 2009;47(14):3210-6. 
Table 1. Participant demographics for all four groups.

\begin{tabular}{|c|c|c|c|c|}
\hline \multirow[b]{2}{*}{ Group } & \multicolumn{2}{|c|}{ mTBI } & \multicolumn{2}{|c|}{ Control } \\
\hline & PCS & None & PCS & None \\
\hline Number of participants & 33 & 73 & 83 & 161 \\
\hline Age $(M \pm S E M) * *$ & $28.2 \pm 2.0$ & $27.4 \pm 1.2$ & $23.5 \pm 0.6$ & $24.0 \pm 0.6$ \\
\hline Gender (F/M) * & $21 / 12$ & $38 / 35$ & $59 / 24$ & $112 / 49$ \\
\hline Education (A/D/P, \%) & $42 / 58 / 0$ & $26 / 63 / 11$ & $23 / 71 / 6$ & $26 / 66 / 8$ \\
\hline Chronic pain (\%) & 9 & 7 & 6 & 2 \\
\hline Other neurological condition (\%) & 3 & 4 & 2 & 1 \\
\hline Medication $(\%){ }^{* * *}$ & 52 & 19 & 23 & 15 \\
\hline Litigation (\%) & 3 & 0 & 1 & 1 \\
\hline Dyslexia (\%) * & 9 & 8 & 2 & 1 \\
\hline IES-R sum score (M士SEM) *** & $19.6 \pm 3.2$ & $7.0 \pm 1.2$ & & \\
\hline Time Since Injury (Years) & $7.8 \pm 1.5$ & $8.5 \pm 0.9$ & & \\
\hline Dizziness (\%) & 100 & 92 & & \\
\hline LOC (\%) & 55 & 51 & & \\
\hline PTA (\%) & 46 & 34 & & \\
\hline Linear/Rotational/Other (\%) & $70 / 12 / 18$ & $78 / 12 / 10$ & & \\
\hline >1 head injury (\%) & 39 & 44 & & \\
\hline Re-injure within 10 days (\%) & 3 & 0 & & \\
\hline \multicolumn{5}{|c|}{ using the chi-square test. $* \mathrm{p}<0.05 ; * * \mathrm{p}<0.01 ; * * * \mathrm{p}<0.001$. Post-hoc analysis of $\chi^{2}$} \\
\hline residuals revealed where the diff & ences in pro & ortion were & een the gr & s (shaded \\
\hline grey box). Education: A: A-Leve & D: Degree, & P: Post-Gra & Degree. I & R: Impact \\
\hline
\end{tabular}


PCS in populations with and without mTBI 34

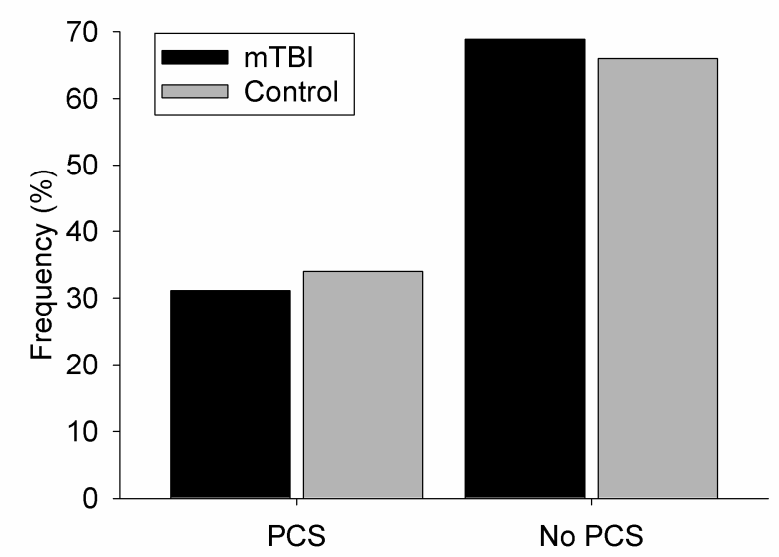

Figure 1. Proportion of participants with PCS, using ICD-10 Diagnosis. 

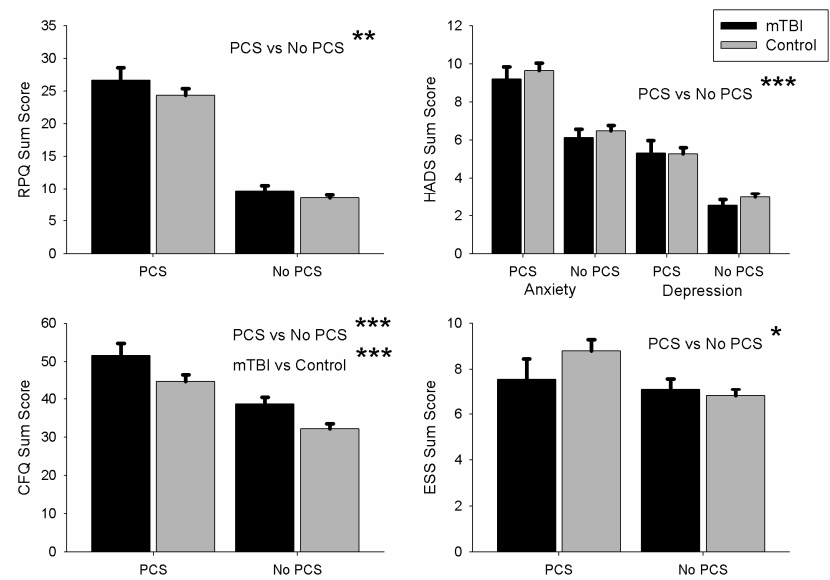

Figure 2. Co-factor scores for all four groups. Top left: Post-Concussion Symptoms (RPQ); top right: Anxiety and Depression (HADS); bottom left: Cognitive Failures (CFQ); bottom right: Daytime Sleepiness (ESS). * $\mathrm{p}<0.05 ; * * \mathrm{p}<0.01 ; * * * \mathrm{p}<0.001$. 


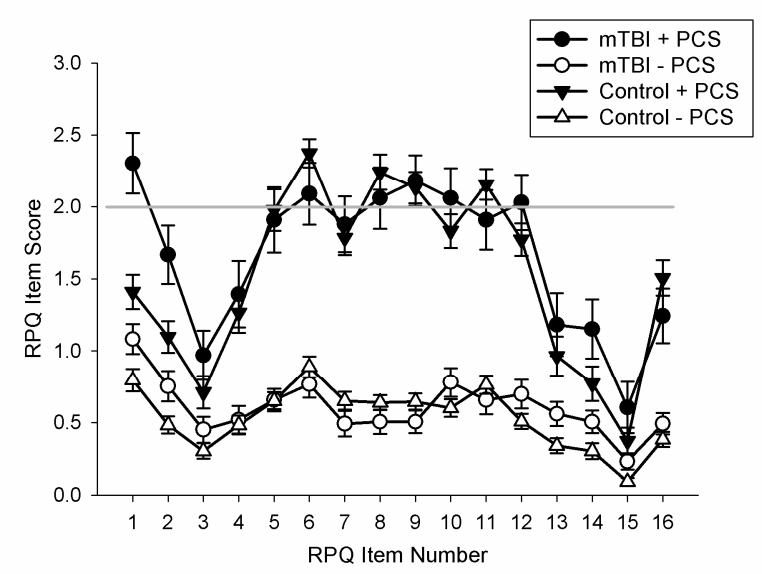

Figure 3. Average RPQ item intensity for the four groups. Grey horizontal line indicates mild symptoms (0: absent, 1: no problem, 2: mild, 3: moderate, 4: severe). The 16 items on the scale are: 1. Headaches; 2. Dizziness; 3. Nausea; 4. Noise sensitivity; 5. Sleep disturbance; 6. Fatigue; 7. Being irritable; 8. Feeling depressed; 9. Feeling frustrated; 10. Poor memory; 11. Poor concentration; 12. Taking longer to think, 13. Blurred vision; 14. Light sensitivity; 15 . Double vision; 16 . Restlessness. 

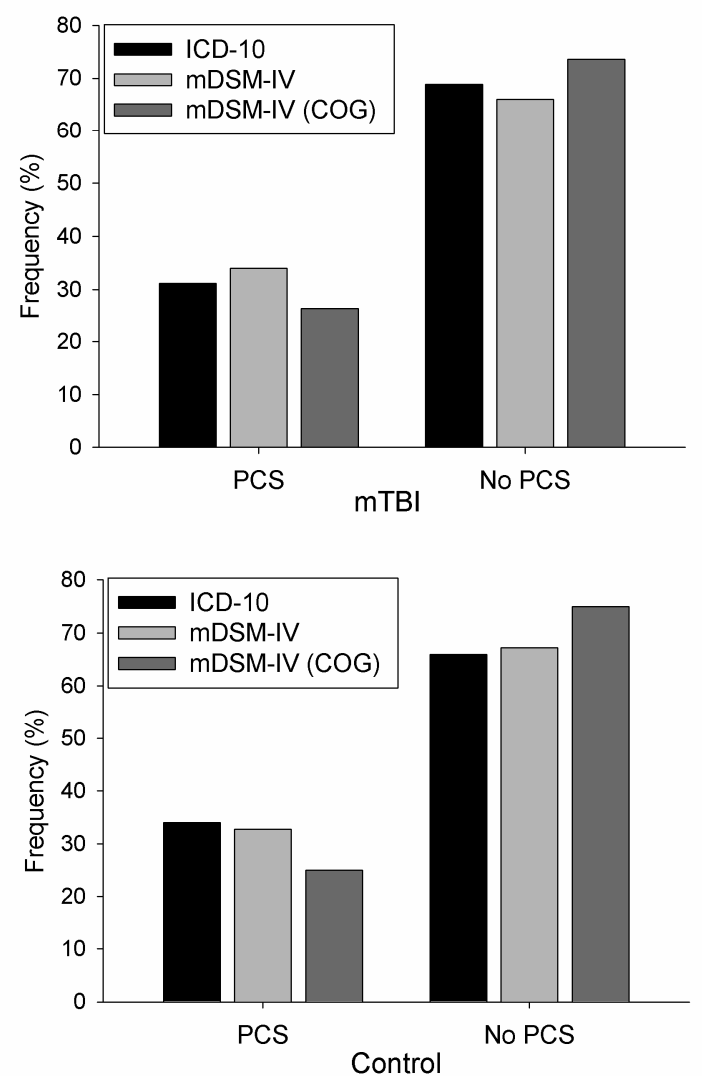

Figure 4. Effect of diagnostic criteria on the proportion of participants with PCS. Top: mTBI group; Bottom: Control group. ICD-10, mDSM-IV and mDSM-IV (COG) diagnostic criteria are used. 